\title{
Dabigatran in the peri-procedural period for radiofrequency ablation of atrial fibrillation: efficacy, safety, and impact on duration of hospital stay
}

\author{
Kimitake Imamura • Akihiro Yoshida • Asumi Takei • \\ Koji Fukuzawa • Kunihiko Kiuchi • Kaoru Takami • \\ Mitsuru Takami • Mitsuaki Itoh • Ryudo Fujiwara • \\ Atsushi Suzuki • Tomoyuki Nakanishi • Soichiro Yamashita • \\ Akinori Matsumoto • Ken-ichi Hirata
}

Received: 31 January 2013 / Accepted: 5 March 2013 / Published online: 14 April 2013

(C) The Author(s) 2013. This article is published with open access at Springerlink.com

\begin{abstract}
Purpose Dabigatran is effective for both the prevention of stroke and bleeding in patients with atrial fibrillation (AF). However, the safety and efficacy of the use of dabigatran in the peri-procedural period for radiofrequency catheter ablation (RFCA) of AF is unknown. Therefore, the purpose of this study was to evaluate the safety and efficacy of dabigatran in the peri-procedural period for RFCA of AF and the duration of hospital stay.

Methods Consecutive patients $(n=227)$ who underwent RFCA for AF were prospectively analyzed. Periprocedural anticoagulant therapy with dabigatran $(n=101$, D group) was compared with warfarin and heparin bridging ( $n=126, \mathrm{~W}$ group). Dabigatran was discontinued 12-24 h before and restarted $3 \mathrm{~h}$ after the procedure. Warfarin was stopped 3 days before the procedure and unfractionated heparin was administered.

Results Ischemic stroke occurred in one patient of the D group $(0.8 \%)$. There was no significant difference between the two groups in the incidence of major bleeding (three cases of cardiac tamponade in each group and one case of intracranial bleeding in the $\mathrm{W}$ group, $p=0.93$ ) or minor bleeding (five cases in the $\mathrm{D}$ group vs. five in the $\mathrm{W}$ group, $p=0.54)$. The
\end{abstract}

K. Imamura $\cdot$ A. Yoshida $(\bowtie) \cdot A$. Takei $\cdot K$. Fukuzawa $\cdot$

K. Kiuchi $\cdot$ K. Takami $\cdot$ M. Takami $\cdot$ M. Itoh $\cdot$ R. Fujiwara

A. Suzuki · T. Nakanishi $\cdot$ S. Yamashita $\cdot$ A. Matsumoto $•$

K.-i. Hirata

Section of Arrhythmia, Division of Cardiovascular Medicine,

Department of Internal Medicine, Kobe University Graduate

School of Medicine, Kobe, Japan

e-mail: ayoshida@med.kobe-u.ac.jp duration of hospital stay was significantly shorter in the D group than in the $\mathrm{W}$ group (7.2 vs. 10.3 days, $p=0.0001$ ). Conclusions Peri-procedural anticoagulation therapy with dabigatran for RFCA of AF was equally safe and effective compared with warfarin and heparin bridging. The use of dabigatran for RFCA of AF shortened the duration of hospital stay.

Keywords Atrial fibrillation - Catheter ablation .

Dabigatran · Complications · Duration of hospital stay

\section{Introduction}

Dabigatran, a new oral direct thrombin inhibitor, has been reported to be safe and effective for the prevention of stroke in patients with atrial fibrillation (AF). Dabigatran $150 \mathrm{mg}$ twice daily reduced both stroke and intracranial and lifethreatening bleeding; dabigatran $110 \mathrm{mg}$ twice daily was not inferior to warfarin for reducing the risk of stroke but significantly reduced intracranial, life-threatening, and major bleeding [1-3]. Based on the results of these clinical trials, it is likely that the frequency of dabigatran use will increase in the future.

It is well known that radiofrequency catheter ablation (RFCA) is an effective treatment for drug-refractory AF, and peri-procedural anticoagulation is particularly important to prevent thromboembolic events [4]. However, the periprocedural anticoagulation protocol varies among centers [5]. Most centers discontinue warfarin (Coumadin) at least 3 to 5 days prior to RFCA and initiate heparin before the procedure as a "bridging strategy." Warfarin is restarted after 
the procedure and heparin is discontinued after therapeutic international normalized ratio (INR) is attained. Recently, some centers have continued warfarin during the procedure to maintain the INR within the therapeutic range [6-8]. Some studies reported that the use of dabigatran in the peri-procedural period for RFCA of AF was effective and safe, but one study reported that peri-procedural dabigatran use increased the risk of thromboembolism and bleeding [9-11]. Thus, the efficacy and safety of dabigatran in the peri-procedural period remains controversial.

It is assumed that the dabigatran would shorten the length of hospital stay compared with the heparin bridging strategy because hospitalization is required at least 3 to 5 days before ablation to initiate heparin therapy in the country where subcutaneous enoxaparin is not available.

In this study, we compared dabigatran with warfarin and heparin bridging in terms of efficacy and safety during the peri-procedural period for RFCA of AF. In addition, we compared the total duration of hospital stay between these two anticoagulation strategies.

\section{Methods}

Between April 2011 and November 2012, 227 consecutive patients who underwent RFCA of AF (165 males, 62 \pm 9 years old, paroxysmal AF $(n=119))$ were prospectively analyzed. Peri-procedural anticoagulation therapy with dabigatran ( $n=101$, D group) was compared with warfarin and heparin bridging ( $n=126, \mathrm{~W}$ group). Patients were divided into two groups based on the discretion of the physician and the hope of patients. The exclusion criteria was age $>80$ years in both groups. In the D group, reasons for exclusion were the presence of severe heart valve disorder. All patients were evaluated for complications related to the procedure at the time of hospitalization and during subsequent follow-up visits up to 1 month after RFCA.

Major bleeding complications were defined as the occurrence of cardiac tamponade, intracranial bleeding, fatal bleeding, any bleeding that resulted in a significant decrease in hemoglobin $(\geq 2 \mathrm{~g} / \mathrm{dl})$ or required transfusion or intervention, or hematoma that required surgery or transfusion. Minor bleeding complications were defined as the occurrence of hematoma or any bleeding that did not require surgery or transfusion.

Thromboembolic complications were defined as the occurrence of ischemic stroke, transient ischemic attack, or peripheral embolic events. Stroke was defined as the sudden onset of a focal neurologic deficit.

The duration of hospital stay was also compared between the two groups.

The distribution of activated partial thromboplastin time (aPTT) values, which provides a useful qualitative assessment of anticoagulant activity [12-14], was analyzed in the $\mathrm{D}$ group during the peri-procedural period.

\subsection{Electrophysiological study and ablation protocol}

All patients had anticoagulation for $\geq 1$ month and transesophageal echocardiography was performed to exclude atrial thrombus before the procedure. All antiarrhythmic medications were stopped four to five half-lives before RFCA, except for amiodarone, which was stopped 1 month before the procedure. Two 8-Fr sheaths, one 8.5-Fr Agilis sheath, one $10.5-\mathrm{Fr}$ sheath, and one 6-Fr sheath were inserted in the femoral vein. One 6-Fr sheath was inserted in the right internal jugular vein or left subclavian vein. Transseptal punctures were performed guided by intracardiac echocardiography (ICE). Pulmonary venous and left atrial angiography was performed and a 3-dimensionsal geometrical map of the atria and pulmonary veins (PVs) was created using the Ensite $\mathrm{NavX}^{\circledR}$ mapping system (St. Jude Medical, Minneapolis, MN). Intravenous heparin was administered immediately after atrial transseptal puncture to maintain an activated clotting time (ACT) of $>300 \mathrm{~s}$. The ACT levels were monitored every $30 \mathrm{~min}$ and additional heparin was injected as necessary to maintain an ACT of $>300$ s. PV mapping was performed with a steerable circular catheter of 20,25, or $30 \mathrm{~mm}$ in diameter (Lasso, Biosense Webster, Diamond Bar, CA). A 3.5-mm-tip irrigation catheter (Coolpath Duo, St. Jude Medical, Minneapolis, MN) or 8-mm-tip ablation catheter (Fantasista, Japan Lifeline, Tokyo, Japan) was selected as the roving and ablation catheter. Encircling PV isolation (PVI) was performed with the NavX mapping system and double-Lasso technique $[15,16]$. The radiofrequency energy output was titrated to 30-35 W along the anterior line and 20-25 W during ablation of the posterior area. After successful isolation of all PVs, high-dose isoproterenol $\left(5-20 \mu \mathrm{gmin}^{-1} \mathrm{~h}^{-1}\right)$ was administered to induce AF. When non-PV foci that could initiate AF were detected, focal ablation was performed. If the non-PV foci originated in the superior vena cava (SVC), segmental isolation of the SVC was performed [17, 18]. After PVI and non-PV foci ablation, burst atrial pacing was performed from four sites: the proximal coronary sinus (CS), distal CS, high right atrium, and left atrial appendage (LAA). Pacing was initiated at a cycle length of $250 \mathrm{~ms}$ and decreased in $10 \mathrm{~ms}$ decrements to the shortest cycle length that allowed 1:1 atrial capture. When typical atrial flutter was induced during burst pacing, cavotricuspid isthmus ablation was performed. We did not perform adjunctive ablation for inducible $\mathrm{AF}$ or atrial tachycardia in paroxysmal AF patients. However, additional ablation lines or ablation of regions with complex fractionated atrial electrograms was performed in persistent AF patients based on the discretion of the operator. 
Light to moderate sedation was used during the procedure. For external cardioversion, transient deep sedation was used.

\subsection{Anticoagulation}

We used two doses of dabigatran (220 or $300 \mathrm{mg} / \mathrm{day})$ depending on the patient's condition. A low dose of dabigatran (220 mg/day) was administered to patients who had one or more of the following conditions: co-administration of potent permeability glycoprotein inhibitors (amiodarone, verapamil, or quinidine) or antiplatelet drugs, renal dysfunction $(\mathrm{CCr}, 30$ $50 \mathrm{ml} / \mathrm{min}$ ), past history of upper gastrointestinal ulcer, or advanced age ( $\geq 70$ years old) [1, 19-23].

The anticoagulation protocol in the peri-procedural period is shown in Fig. 1. In the D group, the patients were admitted 1 day before the procedure and the timing of discontinuation of dabigatran before RFCA was adjusted depending on the thromboembolic risk. Dabigatran was discontinued $24 \mathrm{~h}$ before ablation in patients with paroxysmal $\mathrm{AF}$ and a $\mathrm{CHADS}_{2}$ score of 0 or 1 [24]. In patients with a high thromboembolic risk $\left(\mathrm{CHADS}_{2}\right.$ score of $\geq 2$ or persistent $\left.\mathrm{AF}\right)$, dabigatran was stopped $12 \mathrm{~h}$ before ablation. In the $\mathrm{W}$ group, warfarin was stopped 3 days before RFCA and unfractionated heparin was administered to maintain an aPTT of 2.0 to three times the control value. Heparin was stopped $3 \mathrm{~h}$ before the procedure. In both groups, heparin was administered during the procedure to keep ACT in the range of 300 to $350 \mathrm{~s}$. Immediately after the procedure, heparin was stopped and protamine (20-30 mg) was administered to partially reverse heparin-induced anticoagulation. Then, all sheaths were removed. In the D group, dabigatran was restarted $3 \mathrm{~h}$ after ablation and patients were discharged 3 days after ablation. In the $\mathrm{W}$ group, heparin and warfarin were restarted $3 \mathrm{~h}$ after ablation. After a therapeutic INR was attained, heparin was stopped and the patients were discharged.

\subsection{Follow-up}

All patients were followed up for 1 month before and 1 month after ablation and evaluated for complications. Some patients in the $\mathrm{W}$ group were followed by local doctors to maintain a therapeutic INR. During the 1-month visit after ablation, the recovery progress and any complications including those related to the procedure were reviewed and recorded.

\subsection{Statistical analysis}

All continuous variables are expressed as the mean \pm SD. All categorical variables are reported as the number (percentage) of patients. SPSS version 18.0 (SPSS Inc., Chicago, IL) was used for all statistical analyses. A student's
Fig. 1 This figure displays our algorithm for peri-procedural anticoagulation in patients treated with dabigatran or warfarin and heparin bridging. $P A F$ paroxysmal atrial fibrillation, $A C T$ activated clotting time

\section{【Dabigatran group】}

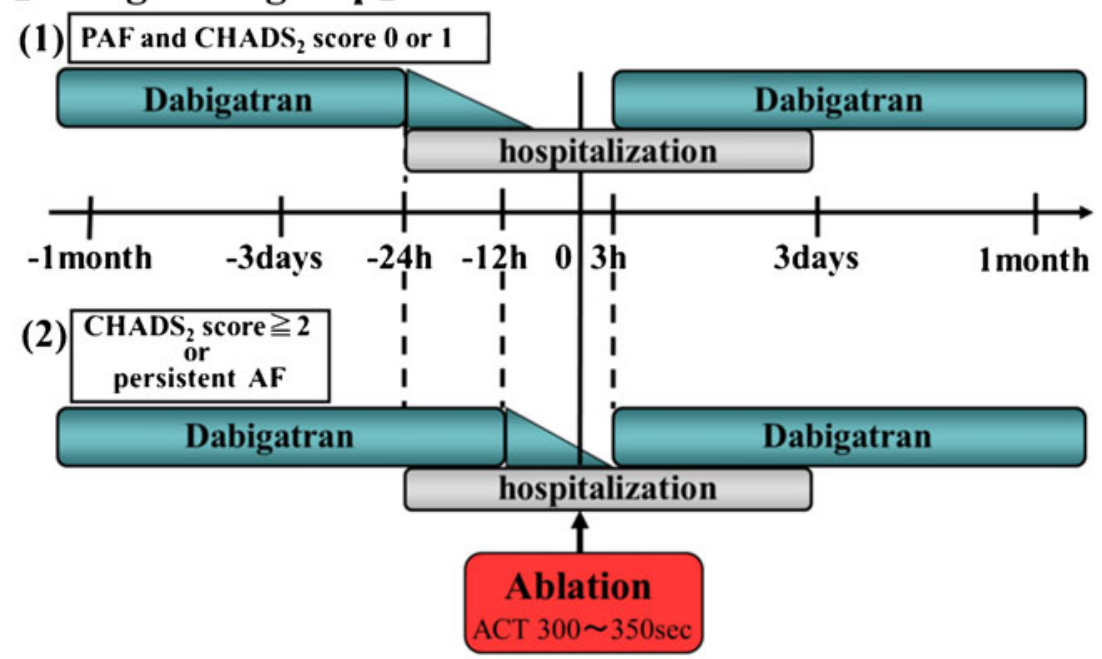

【Warfarin group】

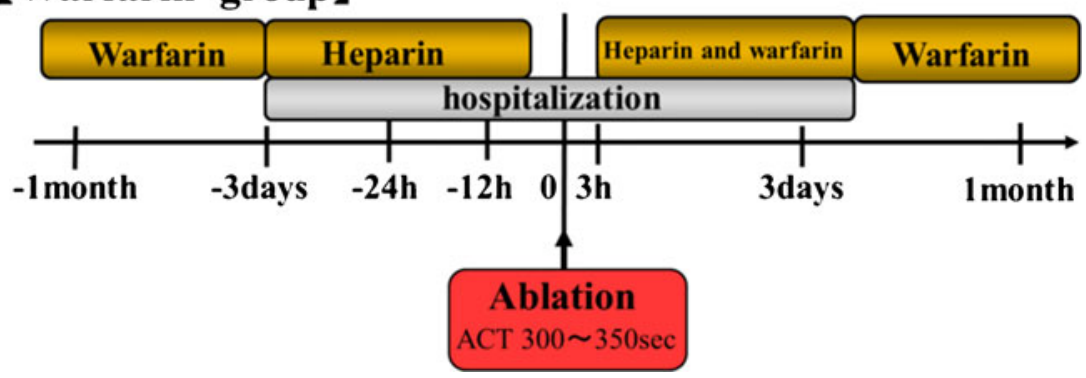


$t$ test was used to compare continuous variables. A $p$ value of $<0.05$ was considered significant.

\section{Results}

\subsection{Clinical and procedural characteristics}

The demographics and clinical characteristics of the patients are summarized in Table 1 . The mean age was $62 \pm 9$ years. Men accounted for $72.7 \%$ of the patients. The AF type was paroxysmal in $119(52.4 \%)$ patients and nonparoxysmal in $107(47.6 \%)$ patients. The $\mathrm{CHADS}_{2}$ score was 0 in 75 (33. $0 \%), 1$ in $105(46.3 \%)$, and $\geq 2$ in $47(20.7 \%)$. There were no differences in the clinical and procedural characteristics between the two groups (Tables 1 and 2).

\subsection{Anticoagulation}

The reason for reducing the dose and the timing of discontinuation of dabigatran before ablation were shown in Table 3 .

We analyzed shifting of the aPTT distribution during the peri-procedural period in the $\mathrm{D}$ group (Fig. 2). The aPTT value at the peak ranged widely from 32 to $73 \mathrm{~s}$ and was $\geq 70 \mathrm{~s}$ in six patients, five of whom were female. The aPTT value at the trough dabigatran levels ranged from 33 to $47 \mathrm{~s}$ before ablation. After dabigatran was discontinued, the aPTT decreased and the mean aPTT just before ablation was 34 s. After dabigatran was restarted, the aPTT value at the trough gradually increased and reached a plateau 3 days after ablation.

Table 1 Clinical characteristics of the patient population

\begin{tabular}{|c|c|c|c|c|}
\hline & All patients $(n=227)$ & D group $(n=101)$ & W group $(n=126)$ & $p$ value \\
\hline Age (years) & $62 \pm 9$ & $61 \pm 10$ & $62 \pm 8$ & 0.33 \\
\hline Male $(n ; \%)$ & $165(72.7)$ & $76(75.2)$ & $89(70.6)$ & 0.46 \\
\hline AF history (months) & $56 \pm 72$ & $48 \pm 59$ & $63 \pm 80$ & 0.10 \\
\hline AF type, paroxysmal $(n ; \%)$ & $119(52.4)$ & $57(56.4)$ & $62(49.2)$ & 0.29 \\
\hline CHADS2 score & $1.0 \pm 0.9$ & $0.9 \pm 0.9$ & $1.1 \pm 1.0$ & 0.22 \\
\hline CHA2DS2-VASc score & $1.7 \pm 1.2$ & $1.6 \pm 1.2$ & $1.8 \pm 1.2$ & 0.11 \\
\hline HAS-BLED score & $0.9 \pm 0.8$ & $0.7 \pm 0.8$ & $1.0 \pm 0.9$ & 0.16 \\
\hline Heart failure $(n ; \%)$ & $38(16.7)$ & $15(14.9)$ & $23(18.3)$ & 0.59 \\
\hline Hypertension $(n ; \%)$ & $121(53.3)$ & $48(47.5)$ & $73(57.9)$ & 0.14 \\
\hline Age $>75$ years $(n ; \%)$ & $8(3.6)$ & $3(3.0)$ & $5(3.9)$ & 0.47 \\
\hline Diabetes mellitus $(n ; \%)$ & $24(10.6)$ & $10(9.9)$ & $14(11.1)$ & 0.83 \\
\hline Prior Stroke/TIA $(n ; \%)$ & $16(7.0)$ & $6(5.9)$ & $10(7.9)$ & 0.61 \\
\hline Coronary artery disease $(n ; \%)$ & $12(5.3)$ & $6(5.9)$ & $6(4.8)$ & 0.77 \\
\hline Antiarrhythmic drugs $(n ; \%)$ & $187(82.4)$ & $83(82.1)$ & $105(83.4)$ & 0.92 \\
\hline Class I group $(n ; \%)$ & $142(62.6)$ & $65(64.4)$ & $77(61.1)$ & 0.45 \\
\hline Bepridil $(n ; \%)$ & $40(17.6)$ & $17(16.8)$ & $23(18.3)$ & 0.86 \\
\hline Amiodarone $(n ; \%)$ & $6(2.6)$ & $1(1.0)$ & $5(4.0)$ & 0.07 \\
\hline Aspirin/antiplatelet drugs $(n ; \%)$ & $17(7.5)$ & $8(7.9)$ & $9(7.1)$ & 0.99 \\
\hline Verapamil $(n ; \%)$ & $48(21.1)$ & $19(18.8)$ & $29(23.0)$ & 0.51 \\
\hline Beta blocker $(n ; \%)$ & $92(40.5)$ & $34(33.7)$ & $58(46.0)$ & 0.07 \\
\hline $\operatorname{ACE}-\mathrm{I} / \mathrm{ARB}(n ; \%)$ & $90(39.6)$ & $36(35.6)$ & $54(42.9)$ & 0.28 \\
\hline Diuretics $(n ; \%)$ & $28(12.3)$ & $9(8.9)$ & $19(15.1)$ & 0.22 \\
\hline Left atrial diameter $(\mathrm{mm})$ & $42 \pm 7$ & $41 \pm 7$ & $42 \pm 7$ & 0.40 \\
\hline LV ejection fraction $(\%)$ & $63 \pm 9$ & $63 \pm 8$ & $62 \pm 9$ & 0.36 \\
\hline Mitral regurgitation & $1.5 \pm 0.7$ & $1.4 \pm 0.6$ & $1.5 \pm 0.7$ & 0.21 \\
\hline Flow velocity in LAA $(\mathrm{cm} / \mathrm{s})$ & $49 \pm 24$ & $51 \pm 26$ & $48 \pm 23$ & 0.36 \\
\hline $\operatorname{SEC}(n ; \%)$ & $37(16.3)$ & $18(17.8)$ & $19(15.1)$ & 0.59 \\
\hline Creatinine $(\mathrm{mg} / \mathrm{dl})$ & $0.8 \pm 0.2$ & $0.8 \pm 0.2$ & $0.8 \pm 0.2$ & 0.42 \\
\hline $\mathrm{CCr}(\mathrm{ml} / \mathrm{min})$ & $88 \pm 22$ & $89 \pm 25$ & $87 \pm 20$ & 0.55 \\
\hline $30-50(\mathrm{ml} / \mathrm{min} ; n ; \%)$ & $4(1.7)$ & $0(0)$ & $4(3.3)$ & 0.19 \\
\hline
\end{tabular}

$A F$ atrial fibrillation, TIA transient ischemic attacks, $L A A$ left atrial appendage, $L V$ left ventricle, $S E C$ spontaneous contrast echo, $C C r$ creatinine clearance 
Table 2 Procedural characteristics in both groups

$R F$ radiofrequency, $P V$ pulmonary vein, $A F L$ atrial flutter

\begin{tabular}{llll}
\hline & D group $(n=101)$ & W group $(n=126)$ & $p$ value \\
\hline Rhythm just before ablation sinus rhythm $(n ; \%)$ & $36(36)$ & $51(40)$ & 0.49 \\
Procedure time (min) & $253 \pm 54$ & $267 \pm 57$ & 0.09 \\
Fluoroscopy time (min) & $94 \pm 22$ & $99 \pm 25$ & 0.13 \\
RF time (min) & $48 \pm 16$ & $52 \pm 17$ & 0.07 \\
Use of irrigation catheter $(n ; \%)$ & $94(90)$ & $108(84)$ & 0.09 \\
Cardioversion during procedure $(n ; \%)$ & $69(68)$ & $94(75)$ & 0.30 \\
Mean number of cardioversion & $1.5 \pm 1.4$ & $1.8 \pm 1.8$ & 0.12 \\
PV isolation $(n ; \%)$ & $101(100)$ & $125(99)$ & 0.98 \\
Non-PV foci ablation $(n ; \%)$ & $8(8)$ & $18(14)$ & 0.15 \\
Common AFL ablation $(n ; \%)$ & $38(38)$ & $40(32)$ & 0.40 \\
Adjunctive therapy $(n ; \%)$ & $11(10)$ & $29(23)$ & 0.55 \\
\hline
\end{tabular}

\subsection{Complications}

Details of complications in the peri-procedural period are shown in Table 4. There were no deaths in this study. A total of $18(7.9 \%)$ patients had either bleeding or thromboembolic complications.

\subsection{Bleeding complications}

Overall bleeding complications occurred in 17 (7.5\%) patients, and seven $(3.1 \%)$ had major bleeding.

The major bleeding complications were six cases of cardiac tamponade that required pericardiocentesis and one case of intracranial bleeding. Pericardiocentesis was performed successfully and hemodynamic function was immediately restored in the majority of patients. No patient required surgery. One patient in each group needed a transfusion due to hypotension from cardiac tamponade. One case of intracranial bleeding occurred before RFCA in the $\mathrm{W}$ group. This

Table 3 The reason for reducing the dose and the timing of discontinuation of dabigatran before ablation

\begin{tabular}{lc}
\hline & D group $(n=101)$ \\
\hline The reason for reducing the dose & $17(17)$ \\
Verapamil $(n ; \%)$ & $1(1)$ \\
Amiodarone $(n ; \%)$ & $7(7)$ \\
Aspirin/antiplatelet $(n ; \%)$ & $0(0)$ \\
Renal dysfunction $(n ; \%)$ & $24(24)$ \\
Age $>70$ years $(n ; \%)$ & $2(2)$ \\
Upper gastrointestinal ulcer & $45(45)$ \\
Total $(n ; \%)$ & $48(48)$ \\
The timing of discontinuation of dabigatran before ablation \\
24 h before ablation $(n ; \%)$ & $53(52)$ \\
12 h before ablation $(n ; \%)$ & \\
\hline
\end{tabular}

patient was a 75 -year-old male who had symptomatic drug refractory paroxysmal AF with heart failure. His $\mathrm{CHADS}_{2}$ score was 2 (congestive heart failure and hypertension), his $\mathrm{CHA}_{2} \mathrm{DS}_{2}$-VASc score was 5 and his HAS-BLED score was 3 (hypertension, elderly, and antiplatelet drugs). Dual antiplatelet therapy (aspirin and clopidogrel) had been initiated for coronary artery disease after implantation of drug-eluting stents. He complained of left hemiparalysis 2 days before the procedure during heparin bridging and was diagnosed with right putaminal hemorrhage by brain plane CT. An emergency craniotomy was performed for removal of the hematoma and continuous ventricular drainage; however, a mild disturbance of consciousness and left hemiparalysis remained.

Most of the minor bleeding events were oozing from the puncture sites in the femoral vein. There was no significant difference in major bleeding complications (3 (3.0\%) events in the $\mathrm{D}$ group vs. 4 (3.2\%) events in the $\mathrm{W}$ group, $p=0.93)$ and minor bleeding (5 (5.0\%) events in the D group vs. 5 $(4.0 \%)$ events in the $\mathrm{W}$ group, $p=0.54)$.

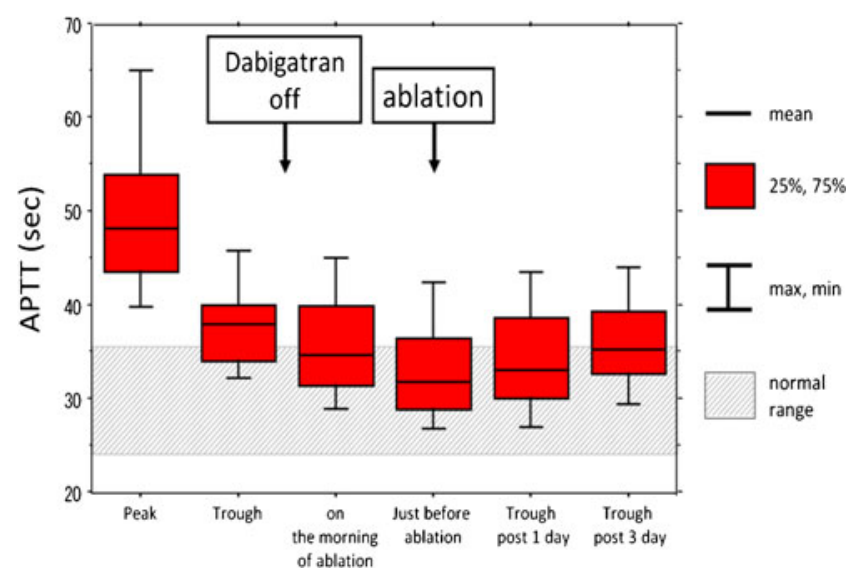

Fig. 2 Shifting of APTT distribution in the dabigatran group. APTT activated partial thromboplastin time 
Table 4 Complications after ablation and duration of hospital stay

D group dabigatran, $W$ group warfarin, TIA transient ischemic attack

\begin{tabular}{llll}
\hline & D group $(n=101)$ & W group $(n=126)$ & $p$ value \\
\hline Thromboembolic complication $(n ; \%)$ & $1(1.0)$ & $0(0)$ & 0.45 \\
Stroke/TIA $(n ; \%)$ & $1(1.0)$ & $0(0)$ & 0.45 \\
Systemic embolism $(n ; \%)$ & $0(0)$ & $0(0)$ & \\
Major bleeding complication $(n ; \%)$ & $3(3.0)$ & $4(3.2)$ & 0.93 \\
Cardiac tamponade $(n ; \%)$ & $3(3.0)$ & $3(2.4)$ & 0.78 \\
Intracranial bleeding $(n ; \%)$ & 0 & $1(0.8)$ & 0.37 \\
Fatal bleeding $(n ; \%)$ & 0 & 0 & 0.54 \\
Minor bleeding complication $(n ; \%)$ & $5(5.0)$ & $5(4.0)$ & 0.33 \\
Others $(n ; \%)$ & $8(7.9)$ & $6(4.8)$ & 0.0001 \\
Duration of hospital stay $($ days $)$ & $7.2 \pm 2.0$ & $10.3 \pm 3.9$ & \\
\hline
\end{tabular}

0001; Table 4). The main reason was the absence of heparin bridging in the peri-procedural period in the $\mathrm{D}$ group.

One thromboembolic complication occurred in the $\mathrm{D}$ group. He was a 73-year-old male with persistent $\mathrm{AF}$, a $\mathrm{CHADS}_{2}$ score of 0 and a $\mathrm{CHA}_{2} \mathrm{DS}_{2}$-VASc score of 1 [25]. His dose of dabigatran was reduced to $220 \mathrm{mg} /$ day considering his advanced age and the co-administration of aspirin due to coronary artery disease. His left atrial diameter was relatively large $(41,77$, and $51 \mathrm{~mm}$ in the parasternal, sagittal, and horizontal echo views, respectively). The flow velocity in the LAA was low $(18 \mathrm{~cm} / \mathrm{s})$ and very strong spontaneous echo contrast was observed by transesophageal echo before ablation and ICE during the procedure. PVI and cavotricuspid isthmus ablation were performed successfully by the irrigated ablation catheter. Right side of medial longitudinal fasciculus syndrome (MLF syndrome: disorder of the coordination and synchronization of all major classes of eye movements and partial unconsciousness) occurred on the next day after the procedure, and multiple cerebral infarctions were suspected based on MRI. Fortunately, his neurologic symptoms resolved completely the day after the event.

\subsection{Other complications}

In the D group, six (5.9\%) patients complained of dyspepsia after starting dabigatran that improved or resolved by oral intake of a proton pump inhibitor without stopping the dabigatran, there was one case of sick sinus syndrome (SSS) that required permanent pacemaker and one case of phrenic nerve palsy. Other complications in the W group included the following: one case of congestive heart failure, one case of SSS that required temporary pacing, two cases of pericarditis, one case of acute paralytic gastric dilatation and one case of urinary tract infection.

\subsection{Duration of hospital stay}

The duration of hospital stay was significantly shorter in the $\mathrm{D}$ group than in the $\mathrm{W}$ group (7.2 \pm 2.0 vs. $10.3 \pm 3.9$ days, $p=0$.

\section{Discussion}

We compared dabigatran with warfarin and heparin bridging in the peri-procedural period for RFCA of AF. There was no significant difference in bleeding complications or thromboembolism between the two groups; however, the use of dabigatran significantly shortened the duration of hospital stay. Dabigatran is an oral direct thrombin inhibitor and its anticoagulant effect in plasma is achieved within $2-3 \mathrm{~h}$ of ingestion. In the RE-LY trial, a dabigatran dose of $300 \mathrm{mg} /$ day was superior to warfarin with regard to stroke prevention in patients with nonvalvular $\mathrm{AF}$, and a $220 \mathrm{mg}$ /day dose of dabigatran was not inferior to warfarin $[1,2]$. The morbidity of $\mathrm{AF}$ is increasing worldwide, and dabigatran is expected to be prescribed more frequently in the future. In this study, we demonstrated the efficacy and safety of dabigatran in the periprocedural period for RFCA of AF.

\subsection{Anticoagulation strategy}

Discontinuation of warfarin 3 to 5 days before RFCA and use of heparin or enoxaparin as a "bridging strategy" is the most frequently used anticoagulation protocol in AF patients undergoing RFCA [4, 26]. Recently, it was shown that continuation of warfarin at a therapeutic INR during the peri-procedural period was effective and safe $[7,8,27]$. The disadvantage of continuation of warfarin is that in cases with major bleeding complications such as cardiac tamponade or intracranial hemorrhage, it takes a long time for vitamin $\mathrm{K}$ to fully antagonize warfarin. In contrast, anticoagulation with heparin is easily induced and can be immediately reversed with protamine. Therefore, heparin bridging is often used in many low-volume centers or by inexperienced operators.

Dabigatran is rapidly absorbed and anticoagulation occurs rapidly within $2-3 \mathrm{~h}$. The mean terminal half-life of 
dabigatran is $8 \mathrm{~h}$ after a single dose and ranges from 12 to 14 $\mathrm{h}$ after multiple doses [28]. Thus, we adjusted the timing of discontinuation of dabigatran before RFCA depending on the thromboembolism risk. Dabigatran was discontinued $24 \mathrm{~h}$ before ablation in the patients with paroxysmal AF and a $\mathrm{CHADS}_{2}$ score of 0 or 1 . In patients with high thromboembolic risk $\left(\mathrm{CHADS}_{2}\right.$ score of $\geq 2$ or persistent $\mathrm{AF}$ ), dabigatran was stopped $12 \mathrm{~h}$ before RFCA, and the procedure was performed after one dabigatran half-life. Dabigatran was restarted $3 \mathrm{~h}$ after ablation. This protocol might reduce bleeding and thromboembolic risk.

In this study, aPTT tests were performed at the time of dabigatran administration (peak), the next morning (trough), just before ablation, and at 1 and 3 days after RFCA. The peak aPTT was $>70 \mathrm{~s}$ in some patients with smaller body weight $(<45 \mathrm{~kg})$ or females. The dose of dabigatran should be reduced in conditions such as the co-administration of potent permeability glycoprotein inhibitors (amiodarone, verapamil, or quinidine), antiplatelet drugs, or renal dysfunction. It was recently reported that high aPTT value correlated with bleeding events [3], and it might be necessary to check the aPTT value before ablation. The dose of dabigatran may need to be reduced in patients with advance age, smaller body weight, or a high aPTT value.

\subsection{Bleeding and thromboembolic complications}

In three previous studies, the incidence of major and minor bleeding complications and thromboembolic complications after RFCA of AF in the peri-procedural period were 0.3 $12.2,0.79-20.0$, and $0.1-1.1 \%$, respectively [7, 27, 29]. The incidence of bleeding and thromboembolism were influenced by various factors, such as patient characteristics, the size of the puncture needle and sheath, the method of transseptal puncture, the value of aPTT during the procedure, the type of ablation catheter, and the RF time [30-33]. In this study, the incidence of major and minor bleeding complications was similar to those in previous studies and were not significantly different between the D and W groups. One thromboembolic event with full recovery occurred in the D group. In this case, the reason for cerebral thromboembolism may have been related to strong spontaneous echo contrast in the LAA, a low LAA flow [32, 34] and persistent AF. The $3 \mathrm{~h}$ delay to the restart of dabigatran after the procedure may have been too long and protamin at the time of removal of the sheaths should not be administrated in such high-risk patients. One case of intracranial bleeding occurred 2 days before RFCA during heparin bridging in the $\mathrm{W}$ group. The $\mathrm{CHA}_{2} \mathrm{DS}_{2}$-VASc score and HAS-BLED score were relatively high in this patient, and the main reason for bleeding was unnecessary triple antithrombotic therapy (aspirin, clopidogrel, and warfarin). Clopidogrel should have been discontinued at least before
RFCA because a drug-eluting stent had been implanted 5 years ago. In cases of cardiac tamponade or excessive bleeding, there is no specific antidote at present for dabigatran. However, the use of fresh frozen plasma, prothrombin complex concentrates, or hemodialysis might be helpful $[32,34]$.

Several studies have been published on the use of dabigatran in the peri-procedural period. Winkle et al. [11] reported that dabigatran is an alternative to anticoagulation with warfarin before and after $\mathrm{AF}$ ablation, but their study had a relatively small sample size. Lakkireddy et al. [10] reported that peri-procedural dabigatran use increases the risk of bleeding and thromboembolic complications. These two previous studies differ from ours in the timing of discontinuation of dabigatran. More recently, Kaseno et al. [9] showed that a $220 \mathrm{mg} /$ day dose of dabigatran was safe for $\mathrm{AF}$ ablation in patients with a relatively low risk of thromboembolism, and their study differs from ours in the dose of dabigatran administered. We adjusted the timing of dabigatran administration (12 or $24 \mathrm{~h}$ ) before RFCA depending on the thromboembolic risk and reduced the dose of dabigatran depending on the patient's condition (co-administration of amiodarone, verapamil antiplatelet drugs, past history of upper gastrointestinal ulcer, or advanced age). This protocol could reduce the risk of bleeding and thromboembolic events.

\subsection{The duration of hospital stay}

In this study, the duration of hospital stay was significantly shorter in the $\mathrm{D}$ group than in the $\mathrm{W}$ group. There are no previous studies that evaluated the duration of hospital stay in RFCA of AF. However, the duration of hospital stay may be not different when RFCA of AF is performed without discontinuation of warfarin and heparin bridging. In the areas where subcutaneous enoxaparin is available in the procedural period of RFCA for AF, the short time hospitalization by the use of dabigatran may not have major advantage compared with heparin bridging strategy. However, the use of dabigatran surrounding AF ablation (stopped 12-24 h before the procedure and restart $3 \mathrm{~h}$ after the procedure) is thought to be more simple and easier methods compared with heparin bridging strategy. There is improved costeffectiveness of dabigatran compared with warfarin [35, 36], and many patients may undergo RFCA of AF under dabigatran in the future.

\subsection{Limitations}

Although there was only one stroke event in the D group, our study was a relatively small observational study, and RFCA was done in only a single center. A larger randomized trial would be required to determine if dabigatran is not 
inferior to warfarin with heparin bridging in the point of thromboembolic risk. Another limitation was that none of the patients in the D group had a low $\mathrm{CCr}(30-50 \mathrm{ml} / \mathrm{min})$. We had no experience with patients who had renal failure in the peri-procedural period.

\section{Conclusions}

Peri-procedural anticoagulation therapy with dabigatran for RFCA of AF was equally safe and effective against warfarin and heparin bridging strategy. The use of dabigatran for $\mathrm{AF}$ in the peri-procedural period shortened the duration of hospital stay. Further studies are needed to evaluate the noninferiority of dabigatran compared with warfarin and heparin bridging strategy.

Open Access This article is distributed under the terms of the Creative Commons Attribution License which permits any use, distribution, and reproduction in any medium, provided the original author(s) and the source are credited.

\section{References}

1. Connolly, S. J., Ezekowitz, M. D., Yusuf, S., Eikelboom, J., Oldgren, J., Parekh, A., et al. (2009). Dabigatran versus warfarin in patients with atrial fibrillation. [Comparative Study Multicenter Study Randomized Controlled Trial Research Support, Non-U.S. Gov't]. The New England Journal of Medicine, 361(12), 11391151.

2. Connolly, S. J., Ezekowitz, M. D., Yusuf, S., Reilly, P. A., \& Wallentin, L. (2010). Newly identified events in the RE-LY trial. [Comment Letter]. The New England Journal of Medicine, 363(19), 1875-1876.

3. Suzuki, S., Otsuka, T., Sagara, K., Matsuno, S., Funada, R., Uejima, T., et al. (2012). Dabigatran in clinical practice for atrial fibrillation with special reference to activated partial thromboplastin time. Circulation Journal, 76(3), 755-757.

4. Calkins, H., Brugada, J., Packer, D. L., Cappato, R., Chen, S. A., Crijns, H. J., et al. (2007). HRS/EHRA/ECAS expert consensus statement on catheter and surgical ablation of atrial fibrillation: recommendations for personnel, policy, procedures and follow-up. A report of the Heart Rhythm Society (HRS) Task Force on Catheter and Surgical Ablation of Atrial Fibrillation developed in partnership with the European Heart Rhythm Association (EHRA) and the European Cardiac Arrhythmia Society (ECAS); in collaboration with the American College of Cardiology (ACC), American Heart Association (AHA), and the Society of Thoracic Surgeons (STS). Endorsed and approved by the governing bodies of the American College of Cardiology, the American Heart Association, the European Cardiac Arrhythmia Society, the European Heart Rhythm Association, the Society of Thoracic Surgeons, and the Heart Rhythm Society. [Practice Guideline]. Europace, 9(6), 335-379.

5. Cappato, R., Calkins, H., Chen, S. A., Davies, W., Iesaka, Y., Kalman, J., et al. (2005). Worldwide survey on the methods, efficacy, and safety of catheter ablation for human atrial fibrillation. [Multicenter Study]. Circulation, 111(9), 1100-1105.

6. Wazni, O. M., Beheiry, S., Fahmy, T., Barrett, C., Hao, S., Patel, D., et al. (2007). Atrial fibrillation ablation in patients with therapeutic international normalized ratio: comparison of strategies of anticoagulation management in the periprocedural period. Circulation, 116(22), 25312534.

7. Hussein, A. A., Martin, D. O., Saliba, W., Patel, D., Karim, S., Batal, O., et al. (2009). Radiofrequency ablation of atrial fibrillation under therapeutic international normalized ratio: a safe and efficacious periprocedural anticoagulation strategy. Heart Rhythm, 6(10), $1425-1429$.

8. Di Biase, L., Burkhardt, J. D., Mohanty, P., Sanchez, J., Horton, R., Gallinghouse, G. J., et al. (2010). Periprocedural stroke and management of major bleeding complications in patients undergoing catheter ablation of atrial fibrillation: the impact of periprocedural therapeutic international normalized ratio. [Comparative Study Multicenter Study Research Support, Non-U.S. Gov't]. Circulation, 121(23), 2550-2556.

9. Kaseno, K., Naito, S., Nakamura, K., Sakamoto, T., Sasaki, T., Tsukada, N., et al. (2012). Efficacy and safety of periprocedural dabigatran in patients undergoing catheter ablation of atrial fibrillation. Circulation Journal, 76(10), 2337-2342.

10. Lakkireddy, D., Reddy, Y. M., Di Biase, L., Vanga, S. R., Santangeli, P., Swarup, V., et al. (2012). Feasibility and safety of dabigatran versus warfarin for periprocedural anticoagulation in patients undergoing radiofrequency ablation for atrial fibrillation: results from a multicenter prospective registry. Journal of the American College of Cardiology, 59(13), 1168-1174.

11. Winkle, R. A., Mead, R. H., Engel, G., Kong, M. H., \& Patrawala, R. A. (2012). The use of dabigatran immediately after atrial fibrillation ablation. Journal of Cardiovascular Electrophysiology, 23(3), 264-268.

12. van Ryn, J., Stangier, J., Haertter, S., Liesenfeld, K. H., Wienen, W., Feuring, M., et al. (2010). Dabigatran etexilate-a novel, reversible, oral direct thrombin inhibitor: interpretation of coagulation assays and reversal of anticoagulant activity. [Review]. Thrombosis and Haemostasis, 103(6), 1116-1127.

13. Liesenfeld, K. H., Schafer, H. G., Troconiz, I. F., Tillmann, C., Eriksson, B. I., \& Stangier, J. (2006). Effects of the direct thrombin inhibitor dabigatran on ex vivo coagulation time in orthopaedic surgery patients: a population model analysis. British Journal of Clinical Pharmacology, 62(5), 527-537.

14. Stangier, J., \& Clemens, A. (2009). Pharmacology, pharmacokinetics, and pharmacodynamics of dabigatran etexilate, an oral direct thrombin inhibitor. [Review]. Clinical and Applied Thrombosis/Hemostasis, 15(Suppl 1), 9S-16S.

15. Ouyang, F., Bansch, D., Ernst, S., Schaumann, A., Hachiya, H., Chen, M., et al. (2004). Complete isolation of left atrium surrounding the pulmonary veins: new insights from the double-Lasso technique in paroxysmal atrial fibrillation. [Evaluation Studies]. Circulation, 110(15), 2090-2096.

16. Ouyang, F., Ernst, S., Chun, J., Bansch, D., Li, Y., Schaumann, A., et al. (2005). Electrophysiological findings during ablation of persistent atrial fibrillation with electroanatomic mapping and double Lasso catheter technique. Circulation, 112(20), 3038-3048.

17. Arruda, M., Mlcochova, H., Prasad, S. K., Kilicaslan, F., Saliba, W., Patel, D., et al. (2007). Electrical isolation of the superior vena cava: an adjunctive strategy to pulmonary vein antrum isolation improving the outcome of AF ablation. [Clinical Trial]. Journal of Cardiovascular Electrophysiology, 18(12), 1261-1266.

18. Yamaguchi, T., Tsuchiya, T., Miyamoto, K., Nagamoto, Y., \& Takahashi, N. (2010). Characterization of non-pulmonary vein foci with an EnSite array in patients with paroxysmal atrial fibrillation. Europace, 12(12), 1698-1706.

19. Eisert, W. G., Hauel, N., Stangier, J., Wienen, W., Clemens, A., \& van Ryn, J. (2010). Dabigatran: an oral novel potent reversible nonpeptide inhibitor of thrombin. [In Vitro Review]. Arteriosclerosis, Thrombosis, and Vascular Biology, 30(10), 1885-1889.

20. Stangier, J., Rathgen, K., Stahle, H., \& Mazur, D. (2010). Influence of renal impairment on the pharmacokinetics and pharmacodynamics 
of oral dabigatran etexilate: an open-label, parallel-group, singlecentre study. [Clinical Trial Research Support, Non-U.S. Gov't]. Clinical Pharmacokinetics, 49(4), 259-268.

21. Eikelboom, J. W., Wallentin, L., Connolly, S. J., Ezekowitz, M., Healey, J. S., Oldgren, J., et al. (2011). Risk of bleeding with 2 doses of dabigatran compared with warfarin in older and younger patients with atrial fibrillation: an analysis of the randomized evaluation of long-term anticoagulant therapy (RE-LY) trial. [Comparative StudyRandomized Controlled TrialResearch Support, Non-U.S. Gov't]. Circulation, 123(21), 2363-2372.

22. Ezekowitz, M. D., Reilly, P. A., Nehmiz, G., Simmers, T. A., Nagarakanti, R., Parcham-Azad, K., et al. (2007). Dabigatran with or without concomitant aspirin compared with warfarin alone in patients with nonvalvular atrial fibrillation (PETRO Study). [Clinical Trial, Phase II Comparative Study Multicenter Study Randomized Controlled Trial Research Support, Non-U.S. Gov't]. The American Journal of Cardiology, 100(9), 1419-1426.

23. Johnson, S. G., Rogers, K., Delate, T., \& Witt, D. M. (2008). Outcomes associated with combined antiplatelet and anticoagulant therapy. Chest, 133(4), 948-954.

24. Gage, B. F., Waterman, A. D., Shannon, W., Boechler, M., Rich, M. W., \& Radford, M. J. (2001). Validation of clinical classification schemes for predicting stroke: results from the National Registry of Atrial Fibrillation. [Research Support, U.S. Gov't, P.H.S. Validation Studies]. Journal of the American Medical Association, 285(22), 2864-2870.

25. Lip, G. Y., Nieuwlaat, R., Pisters, R., Lane, D. A., \& Crijns, H. J. (2010). Refining clinical risk stratification for predicting stroke and thromboembolism in atrial fibrillation using a novel risk factor-based approach: the euro heart survey on atrial fibrillation. [Comparative Study Multicenter Study Research Support, NonU.S. Gov't]. Chest, 137(2), 263-272.

26. Natale, A., Raviele, A., Arentz, T., Calkins, H., Chen, S. A., Haissaguerre, M., et al. (2007). Venice Chart international consensus document on atrial fibrillation ablation. [Consensus Development Conference]. Journal of Cardiovascular Electrophysiology, 18(5), 560-580.

27. Kwak, J. J., Pak, H. N., Jang, J. K., Kim, S. K., Park, J. H., Choi, J. I., et al. (2010). Safety and convenience of continuous warfarin strategy during the periprocedural period in patients who underwent catheter ablation of atrial fibrillation. [Comparative Study Research Support, Non-U.S. Gov't]. Journal of Cardiovascular Electrophysiology, 21(6), 620-625.
28. Hankey, G. J., \& Eikelboom, J. W. (2011). Dabigatran etexilate: a new oral thrombin inhibitor. [Research Support, Non-U.S. Gov't Review]. Circulation, 123(13), 1436-1450.

29. Takahashi, A., Kuwahara, T., \& Takahashi, Y. (2009). Complications in the catheter ablation of atrial fibrillation: incidence and management. [Review]. Circulation Journal, 73(2), 221-226.

30. Prudente, L. A., Moorman, J. R., Lake, D., Xiao, Y., Greebaum, H., Mangrum, J. M., et al. (2009). Femoral vascular complications following catheter ablation of atrial fibrillation. [Controlled Clinical Trial]. Journal of Interventional Cardiac Electrophysiology, 26(1), 59-64.

31. Abhishek, F., Heist, E. K., Barrett, C., Danik, S., Blendea, D., Correnti, C., et al. (2011). Effectiveness of a strategy to reduce major vascular complications from catheter ablation of atrial fibrillation. [Research Support, Non-U.S. Gov't]. Journal of Interventional Cardiac Electrophysiology, 30(3), 211-215.

32. Ren, J. F., Marchlinski, F. E., Callans, D. J., Gerstenfeld, E. P., Dixit, S., Lin, D., et al. (2005). Increased intensity of anticoagulation may reduce risk of thrombus during atrial fibrillation ablation procedures in patients with spontaneous echo contrast. Journal of Cardiovascular Electrophysiology, 16(5), 474477.

33. Scherr, D., Sharma, K., Dalal, D., Spragg, D., Chilukuri, K., Cheng, A., et al. (2009). Incidence and predictors of periprocedural cerebrovascular accident in patients undergoing catheter ablation of atrial fibrillation. [Research Support, Non-U.S. Gov't]. Journal of Cardiovascular Electrophysiology, 20(12), 1357-1363.

34. Zabalgoitia, M., Halperin, J. L., Pearce, L. A., Blackshear, J. L., Asinger, R. W., \& Hart, R. G. (1998). Transesophageal echocardiographic correlates of clinical risk of thromboembolism in nonvalvular atrial fibrillation. Stroke Prevention in Atrial Fibrillation III Investigators. [Research Support, U.S. Gov't, P.H.S.]. Journal of the American College of Cardiology, 31(7), 1622-1626.

35. Kansal, A. R., Sorensen, S. V., Gani, R., Robinson, P., Pan, F., Plumb, J. M., et al. (2012). Cost-effectiveness of dabigatran etexilate for the prevention of stroke and systemic embolism in UK patients with atrial fibrillation. [Comparative Study Multicenter Study Randomized Controlled Trial Research Support, Non-U.S. Gov't]. Heart, 98(7), 573-578.

36. Davidson, T., Husberg, M., Janzon, M., Oldgren, J., \& Levin, L. A. (2012). Cost-effectiveness of dabigatran compared with warfarin for patients with atrial fibrillation in Sweden. European Heart Journal. doi:10.1093/eurheartj/ehs157. 